\title{
Alimentación y reproducción de las principales especies ícticas del río Mesay durante el período de "aguas altas"
}

\author{
María del Pilar Blanco-Parra ${ }^{1}$ \& Ivonne Bejarano-Rodríguez ${ }^{2}$ \\ 1 Posgrado en Ciencias del Mar y Limnología, Universidad Nacional Autónoma de México. Av. Primera 1020 B, \\ Tecnológico, La Paz B.C.S. México, C.P. 23050; pblanco@icmyl.unam.mx \\ 2 Departamento de Ciencias Marinas, Universidad de Puerto Rico. PO Box 3121 Lajas PR 00667; \\ ibejarano@cima.uprm.edu
}

Recibido 20-V-2003. Corregido 01-IX-2005. Aceptado 09-III-2006.

\begin{abstract}
Diet and reproduction of the main fish species from the Mesay river (Colombian Amazon region) during the flooding season. The diet and reproduction of fish communities in three biotopes (river, stream, and lake) of the Mesay floodplain-river complex (Puerto Abeja, Serranía de Chiribiquete National Natural Park, Caquetá, Colombia) were sampled during the "high water" level or flooding period. A total of 79 species of fishes from 15 families and four orders were collected between July and September 2000. The most important items in their diet were fruits and seeds. Approximately $46 \%$ of captured fish were near maturity, and $35 \%$ were mature. The feeding and reproductive behavior of these fish were consistent with other studies on migratory Amazonian species during the high water period, when the floodplain plays an important role in the availability of food and refuge. During this period the fish make use of the vast food availability to accumulate fat reserves that later produce the energy needed for gonadal maturation and breeding migrations. Rev. Biol. Trop. 54 (3): 853-859. Epub 2006 Sept. 29.
\end{abstract}

Key words: Fish, feeding, reproduction, Mesay river, Colombia, Amazonia.

La mayoría de los peces de la región Amazónica cambian su hábitat a lo largo del año en respuesta a las fluctuaciones de los niveles de los ríos. Estos cambios responden a las necesidades tróficas, a la intensidad de depredación y a una estrategia reproductiva; sin embargo otros autores lo atribuyen también a las condiciones fisicoquímicas del agua (Junk 1997). En estos viajes migratorios muchas de las especies recorren largas distancias, como es el caso de los bagres y los carácidos, que para esta área constituyen los principales grupos de peces con migraciones alimentarias (Junk 1997).

El río Mesay corresponde al tipo de aguas negras, según la caracterización hidrográfica de la cuenca amazónica colombiana realizada por el Instituto Geográfico Agustín Codazzi, IGAC (Anónimo 1999). La variación estacional del nivel de las aguas de este río presenta un régimen hidrológico monomodal, con un período de inundación entre abril y septiembre, y uno de estiaje entre diciembre y febrero.

Algunos trabajos sobre la estructura trófica de las comunidades ícticas amazónicas (Knöppel 1969, 1970, Honda 1972, Saul 1975, Gottsberger 1978, Goulding 1979, 1980, Walschburger 1986, Goulding et al. 1988, Walschburger et al. 1990, Silva 1993) resaltan la elevada diversidad de ítems y la abundancia de peces en la cuenca debida en parte a la compleja relación existente entre el ambiente acuático y la planicie inundable.

Este es el primer trabajo de investigación realizado sobre la comunidad íctica del río Mesay. Su principal objetivo fue generar una primera caracterización general de los aspectos tróficos y reproductivos de algunas especies de peces presentes en cuatro diferentes biotopos 
(plano inundable, río, lagos y quebradas) durante el período de inundación del río Mesay en el sector de Puerto Abeja, localizado en el departamento del Caquetá, hacia la zona suroriental del Parque Nacional Natural Serranía de Chiribiquete (PNNSC), Amazonia colombiana.

\section{MATERIALES Y MÉTODOS}

Se realizaron muestreos diarios entre julio y septiembre de 2000 (período de aguas altas del río Mesay), en cinco estaciones de muestreo, cuya localización geográfica se determinó con un geoposisionador satelital (GPS). La estación uno se estableció en el río Mesay, $\left(0^{\circ} 5^{\prime} 6^{\prime \prime} \mathrm{N}, 72^{\circ} 30^{\prime} 56^{\prime \prime} \mathrm{W}\right)$; la estación dos se ubicó en una laguna con un extenso plano de inundación $\left(0^{\circ} 5^{\prime} 27^{\prime \prime} \mathrm{N}, 72^{\circ} 29^{\prime} 34^{\prime \prime} \mathrm{W}\right)$; la estación tres se localizó en la quebrada El Sábalo ( $\left.0^{\circ} 3^{\prime} 55^{\prime \prime} \mathrm{N}-72^{\circ} 28^{\prime} 13^{\prime \prime} \mathrm{W}\right)$; la cuarta estación se ubicó en una laguna pequeña con un plano de inundación pequeño $\left(0^{\circ} 5^{\prime} 6^{\prime \prime} \mathrm{N}, 7^{\circ} 24^{\prime} 55^{\prime}\right.$ ' W); y la estación cinco se estableció en la quebrada Aguanegra (05'9.6” N, 72²8'13” W). Se utilizaron diferentes artes de pesca que incluyeron transmallos con ojo de malla de 11 y $5 \mathrm{~cm}$; y caña y línea de mano con anzuelos número 8 , 12,14 y 16 . En campo no fue posible la estimación de parámetros fisicoquímicos debido a la dificultad logística del traslado de los equipos necesarios para esto, sin embargo se consideraron los datos de estudios previos realizados por el Instituto Geográfico Agustín Codazzi que caracterizan estos parámetros en las aguas de los afluentes de la región amazónica, incluyendo al río Mesay, y los lagos y quebradas de su cuenca. (Anónimo 1999). A todos los ejemplares capturados se les determinó el sexo y su estado de madurez gonadal en campo mediante un análisis macroscópico directo de las gónadas modificado del propuesto por Nikolsky (1963) y Galvis et al. (1989), observándose para cada estado las siguientes características:

- Gónada inmadura, no se evidencia ningún desarrollo, gónada rosada, translúcida, con una longitud de un tercio de la longitud de la cavidad abdominal.

- Madurando, gónadas cerca de un medio de la longitud de la cavidad abdominal, color rosado a amarillo. Testículos en forma de cordón ensanchan anteriormente.

- Gónadas de mayor tamaño, con dos tercios de la longitud de la cavidad abdominal. Color más intenso que en estado $1 \mathrm{y}$ aspecto granular.

- Gónadas maduras, ocupan casi toda la cavidad abdominal, huevos diferenciables a simple vista aunque no salen fácilmente al oprimir el vientre. Testículos color crema.

- Gónadas maduras de gran tamaño, llenan totalmente la cavidad abdominal. Huevos grandes que salen fácil al oprimir el vientre, gónadas color crema y amarillo encendido.

- Gónadas en reposo o recién ovaladas. De aspecto flácido, vacías, con algunos residuos de huevos opacos o esperma.

También se determinó en campo la presencia de cuerpos grasos en la cavidad abdominal de los individuos, y para conocer su dieta se extrajeron los estómagos de 185 individuos pertenecientes a las especies más abundantes. Los estómagos se fijaron en formol al $10 \%$ para posteriormente analizar su contenido en el laboratorio de ictiología del Instituto de Ciencias Naturales de la Universidad Nacional de Colombia (ICN-MHN) en Bogota. Asimismo algunos individuos de las diferentes especies de peces capturadas se fijaron en formol al $10 \%$ y se transportaron al laboratorio para identificarlos a nivel de especie para lo cual se utilizaron las claves propuestas por Eigenmann (1912, 1922), Schultz (1944), Fernández-Yépez (1968) y Géry (1977). Una vez identificados, estos ejemplares se depositaron en la colección ictiológica del ICN-MHN con números de registro del 4246 al 4293 y del 5243 al 5269. 
En el laboratorio se analizó el contenido estomacal de las 29 especies más abundantes. Debido a que el interés era conocer la dieta de la especie y no la de los individuos en particular (Galvis et al. 1989), se formó una muestra por especie en cada biotopo, constituida por los contenidos estomacales de todos los individuos de la especie capturados en éste. El contenido estomacal se examinó bajo el estereoscopio en una caja de Petri, donde se distribuyeron homogéneamente, para su identificación y cuantificación. Las ítems se clasificaron según trabajos previos realizados en la Amazonia como el de Knöppel (1970) y Galvis et al. (1989), y se agruparon en categorías de tipo alóctono y autóctono.

La determinación de contribución de cada ítem en los contenidos estomacales se realizó mediante la metodología de los puntos y la numérica (Marreno 1994). A pesar de tratarse de un método subjetivo es una herramienta útil para obtener una idea de la importancia de los distintos ítems que componen la dieta de cada especie.

Para las especies detritívoras y planctívoras se diluyeron en agua los contenidos estomacales, se agitaron para homogenizar la muestra y con una pipeta plástica se extrajo una alícuota de $2 \mathrm{ml}$, se colocó sobre una lámina portaobjetos y se observó bajo el microscopio compuesto. Los organismos presentes en la muestra se identificaron y cuantificaron. Finalmente se refirieron a una determinación de volumen de cada ítem.

Para saber si había alguna correlación, y por ende, una sobreposición en las dietas de los peces carnívoros, omnívoros y detritívoros se aplicó el coeficiente del ámbito de correlación de Spearman (Krebs 1999).

\section{RESULTADOS}

Se capturaron un total de 5263 peces de 79 especies, pertenecientes a 16 familias y cuatro órdenes.

Durante este período se encontró a un $17 \%$ de los peces examinados con los estómagos vacíos. El $60 \%$ de las especies consumieron tanto ítems autóctonos como alóctonos, el $27 \%$ sólo alóctonos y el $13 \%$ sólo autóctonos. Las especies se dividieron en tres categorías tróficas encontrándose un total de 17 omnívoras, 11 carnívoras y una detritívora. En las especies carnívoras se evidenció un comportamiento atípico en cuanto a sus hábitos alimentarios, ya que tan sólo un $45.5 \%$ de los individuos de esta categoría trófica se alimentaron casi exclusivamente de peces $(95-100 \%$ del contenido estomacal analizado); mientras otro $45.5 \%$ lo hicieron principalmente de frutos y semillas, con porcentajes entre el 70 y $90 \%$ del contenido estomacal analizado; y el $9 \%$ restante basó su dieta en restos vegetales. El grupo de los omnívoros se alimentó principalmente de frutos y semillas (82\%) y en menor medida de otros ítems como restos vegetales (6\%), e insectos terrestres de los órdenes Hymenoptera (6\%) y Orthoptera $(6 \%)$. Los ítems predominantes entre los detritívoros fueron las microalgas chlorophytas encontrándose con valores del $72 \%$ en los contenidos estomacales (Cuadro 1). Durante este período de pulso hídrico del río se encontró que el $98.9 \%$ de todas las especies capturadas presentaron acumulación de cuerpos grasos en la cavidad abdominal. Se encontró una alta correlación (Spearman, $\mathrm{p}<0.05$ ) entre las dietas de los peces carnívoros, omnívoros y detritívoros.

En lo referente a los aspectos reproductivos se observó que durante este período la mayoría de los individuos estaban en etapa de maduración $(46 \%)$, con un $31 \%$ en estado tres y un $15 \%$ en estado dos, seguido de los individuos maduros en estado cuatro (35\%). Los individuos inmaduros (Estados cero y uno, $15 \%$ ) y aquellos que ya habían desovado (Estado cinco, $4 \%$ ) presentaron los menores porcentajes.

Las especies más abundantes, Hemiodus unimaculatus (Bloch, 1794), Leporinus fasciatus (Bloch, 1794), Leporinus brunneus Myers, 1950 y Serrasalmus rhombeus (Linnaeus, 1766), presentaron proporciones de hembras por encima del $70 \%$, con predominancia de individuos maduros en las dos primeras especies y de especímenes en maduración en las 
CUADRO 1

Ítems encontrados en más de un $70 \%$ de los contenidos estomacales de las especies analizadas

TABLE 1

Items found in more than $70 \%$ of the stomach contents for the studied species

\begin{tabular}{|c|c|c|c|}
\hline ESPECIES & $\begin{array}{l}\text { Categoría } \\
\text { Trófica }\end{array}$ & $\begin{array}{c}\text { Número } \\
\text { de } \\
\text { Estómagos }\end{array}$ & Ítem \\
\hline Argonectes scapularis & Omnívora & 11 & $\mathrm{C}$ y H \\
\hline Brycon falcatus & Omnívora & 36 & FS \\
\hline Brycon melanopterus & Omnívora & 4 & FS \\
\hline Brycon opalinus & Omnívora & 1 & $\mathrm{O}$ \\
\hline Catoprion mento & Carnívora & 1 & $\mathrm{P}$ \\
\hline Cichla ocellaris & Carnívora & 2 & $\mathrm{P}$ \\
\hline Curimata ocellata & Detritívora & 1 & $\mathrm{C}$ \\
\hline Hemiodus unimaculatus & Omnívora & 5 & FS y $\mathrm{C}$ \\
\hline Hoplias malabaricus & Carnívora & 1 & RV \\
\hline Hydrolycus scomberoides & Carnívora & 1 & $\mathrm{P}$ \\
\hline Leporinus agassizi & Omnívora & 5 & FS \\
\hline Leporinus brunneus & Omnívora & 7 & FS \\
\hline Leporinus fasciatus & Omnívora & 3 & FS \\
\hline Leporinus klausewitsi & Omnívora & 7 & FS \\
\hline Myleus pacu & Omnívora & 1 & FS \\
\hline Myleus rubripinnis & Omnívora & 4 & FS y RV \\
\hline Parauchenipterus galeatus & Omnívora & 3 & RV, F y H \\
\hline Pimelodus blochii & Omnívora & 10 & FS y $\mathrm{P}$ \\
\hline Platynematichthis notatus & Omnívora & 1 & FS \\
\hline Pristobrycon aureus & Carnívora & 5 & FS \\
\hline Pristobrycon calmoni & Carnívora & 8 & FS \\
\hline Pristobrycon scapularis & Carnívora & 1 & FS \\
\hline Pristobrycon striolatus & Carnívora & 2 & FS \\
\hline Rhaphiodon vulpinus & Carnívora & 1 & $\mathrm{P}$ \\
\hline Serrasalmus rhombeus & Carnívora & 6 & $\mathrm{P}$ \\
\hline Serrasalmus sp. & Carnívora & 4 & FS \\
\hline Triportheus albus & Omnívora & 7 & FS \\
\hline Triportheus angulatus & Omnívora & 3 & FS \\
\hline Triportheus elongatus & Omnívora & 44 & FS \\
\hline
\end{tabular}

FS: Frutos y semillas, P: Peces, C: Chlorophytas, H: Hymenoptera, O: Orthoptera, RV: Restos vegetales, F: Formicoidea, FS: Seeds and fruits, P: Fishes, C: Chlorophytas, H: Hymenoptera, O: Orthoptera, RV: vegetal rests, F: Formicoidea. otras dos. Los machos se encontraron en porcentajes superiores al $70 \%$ en Pristobrycon aureus (Günther, 1864) y Serrasalmus sp., con predominancia de individuos maduros en ambas especies (Cuadro 2).

\section{DISCUSIÓN}

El alto porcentaje (98.9\%) de individuos con acumulación de cuerpos grasos encontrados en este estudio, sumado al bajo porcentaje de individuos con estómagos vacíos (17\%), son una evidencia de que los peces, especialmente los migratorios como Brycon falcatus, B. melanopterus y B. opalinus, acumulan una gran cantidad de grasa a lo largo del período de aguas altas, cuando la oferta alimenticia es abundante y brinda la energía necesaria para el desarrollo gonadal; ya que las migraciones de desove suelen llevarse a cabo en el período de aguas bajas cuando la disponibilidad de alimento es limitada (Junk 1997). Sin embargo, esto no sólo depende de la cantidad de alimento disponible sino también de la condición del pez, pues muchas especies de peces dejan de alimentarse durante sus períodos de desove (Junk 1985). Lo anterior puede explicar lo observado en la mayoría de los individuos de Cichla ocellaris que presentaron estómagos vacíos o con cantidades mínimas de alimento, posiblemente asociadas a un estado de madurez gonadal avanzado, pues el $59 \%$ de los individuos capturados de esta especie se encontraron en estado cuatro de madurez gonadal. Aunque no se puede descartar que esta ausencia de alimento se deba a la rápida digestión de este.

La mayoría de los individuos a los que se les analizó el contenido estomacal, se estaban alimentando de los recursos provistos por el bosque (alóctonos), debido a que en la estación de aguas altas la mayor parte del alimento es producido en el plano inundado proveniente de la vegetación arbórea inundada y del bosque no inundable circundante representado principalmente por insectos, artrópodos y la máxima producción de frutos y semillas que quedan 
CUADRO 2

Porcentajes de hembras y machos en los diferentes estados de madurez para las especies más abundantes

TABLE 2

Percentage of females and males in the maturity stages for the more abundant species

\begin{tabular}{lccccccccc} 
& \multicolumn{4}{c}{ Sexo } & \multicolumn{7}{c}{ \% Estados Madurez } \\
Especies & No. Ind & H\% & M\% & $\mathbf{0}$ & $\mathbf{1}$ & $\mathbf{2}$ & $\mathbf{3}$ & $\mathbf{4}$ & $\mathbf{5}$ \\
Argonectes scapularis & 32 & 63 & 37 & 0 & 3 & 6 & $\mathbf{2 8}$ & $\mathbf{5 9}$ & 3 \\
Brycon falcatus & 137 & 55 & 45 & 4 & 13 & 15 & $\mathbf{3 4}$ & $\mathbf{3 4}$ & 1 \\
Cichla ocellaris & 32 & 47 & 53 & 0 & $\mathbf{2 8}$ & 0 & 13 & $\mathbf{5 9}$ & 0 \\
Hemiodus unimaculatus & 33 & 82 & 18 & 0 & 0 & 6 & 12 & $\mathbf{7 0}$ & 12 \\
Leporinus brunneus & 33 & 88 & 12 & 6 & 3 & 6 & $\mathbf{4 5}$ & $\mathbf{3 9}$ & 0 \\
Leporinus fasciatus & 10 & 80 & 20 & 0 & 0 & 10 & 10 & $\mathbf{7 0}$ & 10 \\
Leporinus klausewitzi & 17 & 35 & 65 & 0 & 12 & 24 & $\mathbf{2 9}$ & $\mathbf{3 5}$ & 0 \\
Myleus rubripinnis & 32 & 47 & 53 & 3 & 6 & 23 & $\mathbf{2 9}$ & $\mathbf{3 9}$ & 0 \\
Pimelodus blochii & 69 & 65 & 35 & 4 & 20 & $\mathbf{2 3}$ & $\mathbf{3 3}$ & 19 & 0 \\
Pristobrycon aureus & 11 & 27 & 73 & 18 & 0 & 18 & 18 & $\mathbf{4 5}$ & 0 \\
Pristobrycon calmoni & 37 & 59 & 41 & 6 & 25 & 17 & $\mathbf{3 6}$ & 17 & 0 \\
Pristobrycon striolatus & 13 & 62 & 38 & $\mathbf{2 3}$ & $\mathbf{2 3}$ & $\mathbf{2 3}$ & $\mathbf{2 3}$ & 8 & 0 \\
Semaprochilodus amazonensis & 13 & 62 & 38 & 0 & 0 & 8 & 8 & $\mathbf{7 7}$ & 8 \\
Serrasalmus rhombeus & 12 & 83 & 17 & 0 & 8 & 0 & $\mathbf{5 0}$ & $\mathbf{4 2}$ & 0 \\
Serrasalmus sp. & 15 & 7 & 93 & 13 & 0 & 7 & $\mathbf{2 0}$ & $\mathbf{6 0}$ & 0 \\
Triportheus albus & 65 & 40 & 60 & 0 & 12 & 15 & $\mathbf{4 3}$ & $\mathbf{2 6}$ & 3 \\
Triportheus angulatus & 32 & 34 & 66 & 0 & 3 & 6 & $\mathbf{3 4}$ & $\mathbf{5 3}$ & 3 \\
Triportheus elongatus & 62 & 38 & 7 & 22 & $\mathbf{2 9}$ & $\mathbf{2 3}$ & 18 & 2
\end{tabular}

H: hembras M: machos No. Ind: número de individuos. H: Females M: males No. Ind: number of individuals.

disponibles en el agua como un potencial recurso alimenticio para los peces.

El comportamiento alimentario de la mayoría de las especies carnívoras encontradas en este estudio mostró que en esta época los individuos basan su dieta no sólo en los ítems que los ubican en su categoría trófica, sino que también se alimentan de manera importante de frutos y semillas que pertenecen a otro tipo de dietas como las de los omnívoros, ya que la mayor variabilidad de la oferta alimenticia hace que las especies aprovechen un mayor ámbito de ítems (Nikolsky 1963).

El grupo de los omnívoros presentó una preferencia por los ítems de tipo alóctono, entre los que predominan los frutos y semillas.
Esto está relacionado con la gran oferta que el bosque inundado brinda en esta época a los organismos acuáticos. Por la gran variedad de ítems consumidos por estas especies se puede decir que presentan una estrategia alimentaria generalista, lo que indicaría una amplitud de nicho trófico grande.

Curimata ocellata fue la única especie detritívora a la que se le analizó el contenido estomacal. Sin embargo, otras especies consideradas omnívoras, como Argonectes scapularis, Böhlke y Myers 1956 y H. unimaculatus, consumieron ítems tanto de superficie (frutos y semillas) como de fondo (detrito). Knöppel (1970) atribuye la presencia de ítems de superficie y de fondo en un mismo estómago 
a la capacidad de los peces de presentar una alimentación variada cuando la oferta es elevada. El análisis de la composición algal del detrito permite dar una idea general de la composición del fitoplancton encontrado en la columna de agua y éstas a su vez, según Margalef (1983), reflejan fielmente las condiciones particulares de un ecosistema acuático en un momento dado. Así, por ejemplo, la predominancia de clorófitas desmidiáceas (72\%) en los contenidos estomacales analizados en este estudio, se asocia a condiciones de oligotrofía, aguas con tendencia a un $\mathrm{pH}$ ácido, poca mineralización y posible presencia de materia orgánica (Pinilla 1998); lo cual es típico de las aguas negras en esta época en que el aumento del medio acuático diluye los pocos nutrientes que se encuentran en el agua y la materia orgánica que provee el bosque inundado queda disponible.

Es importante resaltar que los contenidos estomacales de los diversos grupos de peces analizados fueron muy similares encontrándose una alta correlación entre el grupo de los carnívoros, omnívoros y detritívoros, mostrando una uniformidad en las dietas de dichos individuos durante el período de inundación, independiente de la categoría trófica a la que pertenecían las diferentes especies, lo que indica una flexibilidad y denota un carácter oportunista de esta ictiofauna en sus dietas.

El mayor porcentaje (66\%) de individuos maduros o en maduración encontrados durante este estudio parece estar relacionado con el aumento del hábitat acuático y la disponibilidad de recursos alimenticios que brindan un ambiente favorable para la reproducción de los peces, además de ofrecer lugares adecuados para la puesta y eclosión de los huevos y refugio para los alevinos. Además la mayoría de peces migratorios de la Amazonia desovan una vez al año, normalmente durante el período de aguas altas. Esta estrategia de reproducción requiere de grandes cantidades de energía para el desarrollo gonadal, la migración y el desove cuando la comida escasea y los peces son muy activos (Junk 1997). Sin embargo, muchas de las especies no migratorias, por ejemplo cíchlidos y scíaenidos, se reproducen varias veces al año, por lo que se les puede capturar en diferentes estados gonadales sin importar el nivel de las aguas (Junk 1997). Tal es el caso de $C$. ocellaris que se presentó con un gran número de individuos tanto maduros (estado cuatro) como inmaduros (estado uno).

\section{AGRADECIMIENTOS}

Las autoras agradecen a la Fundación Puerto Rastrojo y al Centro de Investigaciones Científicas de la Universidad de Bogotá Jorge Tadeo Lozano por el apoyo financiero y logístico. Al Instituto de Ciencias Naturales de la Universidad Nacional de Colombia, por permitir el uso de sus instalaciones durante la fase de laboratorio. A José Iván Mojica, Germán Gálvis, Jaime Díaz, Arturo Acero y Felipe Galván por su asesoría a lo largo de este trabajo.

\section{RESUMEN}

Se describen aspectos alimentarios y reproductivos de la comunidad íctica presente durante el período de "aguas altas", en tres biotopos (río, quebrada y lago) del río Mesay, en el Parque Natural Nacional Serranía de Chiribiquete, Caquetá, Colombia. Se identificaron un total de 79 especies, pertenecientes a 15 familias y cuatro órdenes, recolectados entre julio y septiembre de 2000. El ítem más importante en la dieta de los peces analizados fue el de frutos y semillas. Un $46 \%$ de los peces estaban en etapa de maduración y el $35 \%$ eran maduros. El comportamiento alimentario y reproductivo de la ictiofauna capturada es el típico encontrado en otros estudios para especies amazónicas migratorias durante el período de inundación, cuando la planicie de inundación cumple un papel fundamental en cuanto a la oferta de alimento y refugio; los peces aprovechan la gran disponibilidad de alimento para acumular reservas de grasa que posteriormente les aportarán la energía necesaria para la maduración de las gónadas y las migraciones reproductivas.

Palabras clave: Peces, alimentación, reproducción, río Mesay, Colombia, Amazonia.

\section{REFERENCIAS}

Anónimo. 1999. Paisajes fisiográficos de OrinoquiaAmazonia (ORAM) Colombia. Análisis Geográficos IGAC, Bogotá, Colombia. 120 p. 
Eigenmann, C. 1912. The fresh water fishes of British Guyana, including a study of species and the relations of the fauna of the plateau to that of the lowlands. Mem. Carneige Mus. 5. 578 p.

Eigenmann, C. 1922. The fishes of Northwestern South America, including Colombia, Panamá and the Pacific slopes of the Ecuador and Perú, together with an appendix upon the fishes of the río Meta in Colombia. Mem. Carneige Mus. 5: 1-346.

Fernandez-Yepez, A. 1968. Contribución al conocimiento de la familia Doradidae en Venezuela. Bol. Inst. Oceanogr. Univ. Oriente. 7: 7-72.

Galvis, G., J.I. Mojica \& F. Rodríguez. 1989. Estudio ecológico de una laguna de desborde del río Metica. Fondo FEN Colombia, Universidad Nacional de Colombia, Bogotá, Colombia. 110 p.

Gery, J. 1977. Characoids of the world. T.F.H., Neptune City, Nueva Jersey, EEUU. 672 p.

Gottsberger, G. 1978. Seed dispersal by fish in the inundable region of Humaita, Amazonas. Biotrópica 10: 163-170.

Goulding, M. 1979. Ecología da pesca do rio Madeira. INPA/CNPq. Manaos, Brasil. 172 p.

Goulding, M. 1980. The fishes and the forest. Explorations in the Amazonian natural history. Univ. California, Los Ángeles, California, EEUU. 280 p.

Goulding, M., M. Carvalho \& E.G. Ferreira. 1988. Río Negro, rich live in poor water. SBP, La Haya, Holanda. 200 p.

Honda, E.M. 1972. Contribuçao ao conhecimiento da biología da peixes da Amazonas: I-Alimentaçao de Geophagus. Acta Amazon. 2: 81-88.

Junk, W.J. 1985. Temporary fat storage, an adaptation of some fish species to the waterlevel fluctuations and related environmental changes of the Amazon river. Amazoniana 3: 315-351.

Junk, W.J. 1997. The central Amazon floodplain. Ecology of a pulsing system. Springer, Heidelberg, Alemania. 525 p.

Knöppel, H. 1969. Investigaçoes acerca da agua clara amazónico, baseados no contenudo estomacal de
Gymnotus carapo L. E Pyrrhulina brevis. In II Simposio y Foro de Biología Tropical Amazónica. Idrobo, Florencia, Caquetá, Colombia. 70-78.

Knöppel, H. 1970. Food of central Amazonian fishes, contribution to the nutrient ecology of Amazonian rain-foreststream. Amazoniana 2: 257-352 .

Krebs, C.J. 1999. Ecological methodology. AddisonWesley, Menlo Park, California, EEUU. p. 383-395.

Margalef, R. 1983. Limnología. Omega, Barcelona, España. 1010 p.

Marrero, C. 1994. Métodos para cuantificar contenidos estomacales en peces. Universidad Nacional Experimental de los Llanos Occidentales Ezequiel Zamora, Caracas, Venezuela. 50 p.

Nikolsky, G.V. 1963. The ecology of fishes. Academic, Londres. 352 p.

Pinilla, G.A. 1998. Indicadores biológicos en ecosistemas acuáticos continentales de Colombia: compilación bibliográfica. Fundación Universidad de Bogotá Jorge Tadeo Lozano, Bogotá, Colombia. 67 p.

Saul, W.G. 1975. An ecological study of fishes at a site in upper Amazonian, Ecuador. Proc. Acad. Nat. Sci. Philadelphia. 127: 93-134.

Schultz, P.L. 1944. The fishes of the family Characidae from Venezuela, with descriptions of seventeen new forms. Proc. U.S. Nat. M. 95: 235-367.

Silva, C. 1993. Alimentaçao e distribuçao espacial de algumás espécies de peixes do Igarapé do Candirú, Amazonas, Brasil. Acta Amazon. 23 : 271-285.

Val, A.L. \& V.M.F. de Almeida-Val. 1995. The fishes of the Amazon and their Environment: physiological and biochemical aspects. Springer, Berlín, Alemania. $224 \mathrm{p}$.

Walschburger, T. 1986. Los peces y el bosque. Amazonía última frontera. INDERENA 1: 13-16.

Walschburger, T., C. Monje \& D. Muñoz.1990. Importancia de los bosques inundables para el recurso pesquero en la Amazonía colombiana. Fundación Puerto Rastrojo. COLCIENCIAS, TROPEMBOS, Bogotá, Colombia. 400 p. 
